\title{
Eosinophilic cystitis in children: A case report
}

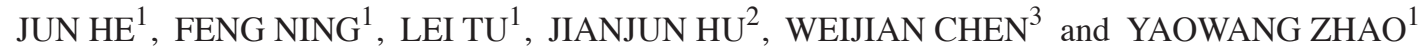 \\ Departments of ${ }^{1}$ Urology, ${ }^{2}$ Radiology and ${ }^{3}$ Pathology, Hunan Children's Hospital, Changsha, Hunan 410007, P.R. China
}

Received December 5, 2017; Accepted May 11, 2018

DOI: $10.3892 /$ etm.2018.6578

\begin{abstract}
The aim of the present case report was to investigate the clinical features, pathological examination and treatment of eosinophilic cystitis (EC) in children. Two cases of EC were reported and reviewed from January 2016 to March 2017. Case 1 (male; 6 years old) had intermittent hematuria, frequent urination, urgent urination, difficulty in urination and abdominal pain. Case 2 (male; 7 years old) had frequent urination, urgent urination, urinary pain, dysuria and suprapubic pain with no hematuria. One patient had a history of allergies and both patients underwent a cystoscope biopsy. Blood eosinophils were clearly increased and a bone marrow biopsy examination revealed that marrow eosinophils were also increased in both cases. The urine culture results were negative. Ultrasonography and computed tomography revealed uneven thickening of the bladder wall and diffusive mucosal lesions. Cystoscopy revealed that the bladder volume became smaller and the mucosa at the bladder floor and neck was red. Lesions were biopsied through the urethra and the following characteristics were observed: Congestion and edema of the bladder mucosa, infiltration of the blood vessels and eosinophils in the muscular layer, accompanied by focal muscle necrosis. Patient 1 was administered anti-inflammatory and cetirizine hydrochloride treatments, followed by 6 weeks of prednisone dose-reduction therapy. Patient 2 was administered antibiotics and cetirizine hydrochloride. Following 6-month follow-ups, abnormal voiding symptoms had disappeared in each case. Ultrasonography and computed tomography revealed no bladder wall thickening or space-occupying lesions. EC in children is rare and easily misdiagnosed as nonspecific bladder inflammation or bladder occupying lesions. Cystoscopy and biopsy are necessary to diagnose EC and conservative treatments with anti-inflammatory, anti-allergic and cortical hormone nonspecific treatments are suggested.
\end{abstract}

Correspondence to: Dr Yaowang Zhao, Department of Urology, Hunan Children's Hospital, 86 Ziyuan Road, Changsha, Hunan 410007, P.R. China

E-mail: hjys840808@163.com

Key words: cystitis, eosinophilic, child, clinical, treatment

\section{Introduction}

Eosinophilic cystitis (EC) is a type of cystitis with unknown etiology, which was first reported by Brown et al (1) in 1960 (2). The disease rarely occurs in children and, to the best of our knowledge, only 59 cases have been previously reported. As for children, adults are rarely affected by EC. Incidence in males is larger compared with females and onset develops at a mean age of $6.0 \pm 0.5$ years $(3,4)$. EC has been reported to be local manifestations of systemic 'hypereosinophilic' syndromes and allergic diseases, and is associated with bladder trauma, infections and certain medications, including iodine or anesthetic ointments $(5,6)$. The clinical manifestations of EC include hematuria, irritative voiding symptoms and bladder wall thickening, which is often misdiagnosed as tumors (7). The pathological manifestation of EC includes eosinophilic infiltration into layers of the bladder wall (4). EC may be caused by immune system regulation disorders, which induce antigen stimulation and lead to immunoglobulin E (IgE)-mediated eosinophil activation and degranulation of mast cells, triggering the release of inflammatory mediators and injuring the bladder wall (8). Adult patients with EC may present with a variety of urinary symptoms, including frequent urination, urgent urination, odynuria, hematuria, dysuria and suprapubic pain; children with EC often have similar symptoms $(9,10)$. Pathologic biopsy findings are therefore paramount for differential diagnosis (11). Treatments for EC include observation, identification of allergens, antibiotics, antihistamines and steroids (12). For intractable hematuria, transurethral resection of bladder lesions and partial cystectomy may also be used (8). The current study presents two cases of EC, which were diagnosed and treated at the Children's Hospital of Hunan (Changsha, China) between January 2016 and March 2017. Clinical manifestations, pathological features, corresponding treatments and follow-ups are reported.

\section{Case report}

Case 1. Patient 1 was a 6-year-old male who first presented with intermittent gross hematuria, frequent urination, urgent urination, odynuria and abdominal pain in January 2016 at the Children's Hospital of Hunan (Changsha, China). The frequency of urination reached 30 times a day, however the increased rate of urination was primarily at night. Urination was difficult, although the patient was able to form a stream. The patient had no history of hemorrhage or allergy and no lumbago or fever on admission (Table I). The patient 
was diagnosed as having a urinary infection and given one week of anti-inflammatory treatment (Cefmetazole, $100 \mathrm{mg} / \mathrm{kg} / \mathrm{d}$ ) two days prior to the aggravation of dysuresia symptoms. Ultrasonography revealed a thickened bladder wall. Following anti-inflammatory treatment (Cefmetazole, $100 \mathrm{mg} / \mathrm{kg} / \mathrm{d}$ ) for further 3 days, the symptoms disappeared. The patient had normal blood pressure on admission and routine blood tests revealed eosinophil ratios of 24.6, 33.0 and $29.4 \%$ (normal range, $0.5-5 \%$ ) (13) on day 1 , at the end of week 1 and at the end of week 2 , respectively. In addition, the red blood cell count and hemoglobin were observed using peripheral blood smears (data not shown); all results were normal. During hospitalization, urine samples were collected by urinary bladder catheterization and sent to the hospital laboratory. Urine cultures were prepared on blood agar medium and MacConkey agar (PlastLabor Microbiologica, Rio de Janeiro, Brazil). A Vitek (bioMérieux, Marcy-l'Étoile, France) was used to identify and test the susceptibility of strains. A urinary tract infection was established at $\geq 50,000$ colony forming units $/ \mathrm{ml}$ of a single microbial growth. These urine culture tests yielded negative results and a routine urine test and microscopy revealed 20-25 red blood cells in each high-power field of view (data not shown). The specific gravity of urine was 1.025 and a urine nitrite test was negative (data not shown). Renal functions were normal and the concentration of complement C3 was $122 \mathrm{mg} / \mathrm{dl}$. A tuberculin test had negative results and urine polymerase chain reaction (PCR) revealed no adenovirus or Mycobacterium tuberculosis (data not shown). Levels of Dermatophagoides pteronyssinus (D1) and Dermatophagoides culinae (D2) allergens were grade 2 (14). Ultrasonography indicated obvious thickening and abundant blood flow in the bladder wall (Fig. 1A and B). Computed tomography revealed local mucosal lesions with thickening of the right and posterior wall of the bladder (Fig. 2A-D). Retrograde angiography of the bladder determined that the bladder volume decreased and the bladder wall was irregular (Fig. 3A and B). Cystoscopy also revealed that the bladder volume became smaller and the mucosa at the bladder floor and neck became red (data not shown). Based on the above examinations, rhabdomyosarcoma of the bladder was initially considered as a diagnosis. Biopsy of the lesions through the urethra was performed and specimen were fixed at room temperature in $10 \%$ formalin for $12-24 \mathrm{~h}$, embedded in paraffin at $62^{\circ} \mathrm{C}$ for $3 \mathrm{~h}$, cut into $4-\mu \mathrm{m}$-thick sections and stained with a hematoxylin (Shanghai Regal Biology Technology Co., Ltd., Shanghai, China) and eosin (Sinopharm Chemical Reagent Co., Ltd., Shanghai, China) according to the manufacturer's protocol. Specimens were examined histopathologically using a light microscope (magnification, x400; DM 2000 LED; Leica Microsystems, Inc., Buffalo Grove, IL, USA) to assess the types of lesions (15). Lesions revealed congestion and edema of the bladder mucosa (Fig. 4) and infiltration of the blood vessels and eosinophils into the muscular layer (Fig. 5) accompanied by focal muscle necrosis (Fig. 6). A bone marrow biopsy demonstrated eosinophilia (Fig. 7; Table II). Following diagnosis, the patient was given anti-inflammatory (Cefmetazole, $100 \mathrm{mg} / \mathrm{kg} / \mathrm{d}$ ) and cetirizine hydrochloride $(1 \mathrm{ml} / \mathrm{day})$ treatments for a week, followed by 6 weeks of prednisone dose-reduction therapy (16). One month later, re-examination revealed that the symptoms had disappeared and imaging results were normal (Fig. 8A and B). The blood eosinophil content was $1.19 \%$. Following continued intake of low-dose prednisone ( $5 \mathrm{mg} /$ day) for 2 months, the level of blood eosinophils was normal. Imaging examination also revealed completely normal results (Fig. 9A and B; Table III).

Case 2. Patient 2 was a 7-year-old male who presented with acute dysuria, suprapubic pain, frequent urination, urgent urination, urgent incontinence and odynuria. The patient had no history of allergies and had normal body development (Table I). On admission in March 2017, the patient had a normal blood pressure and the eosinophil ratios were $7.9,5.6$ and $0.09 \%$ on day 1 , at the end of week 1 and at the end of week 2, respectively. Normal red blood cells and normal hemoglobin were observed in the peripheral blood smears (data not shown). A urine culture test yielded negative results, while a routine urine test and microscopy revealed no abnormalities (data not shown). The specific gravity of the urine was 1.030 and a nitrite test in the urine yielded negative results (data not shown). The patient's renal functions were normal and the concentration of complement C3 was $109 \mathrm{mg} / \mathrm{dl}$. PPD experiments yielded negative results and urine PCR revealed no adenovirus or Mycobacterium tuberculosis (data not shown). No clear allergens were observed in the allergen screening (data not shown). Computed tomography and retrograde angiography of the bladder revealed local mucosal lesions with thickening of each side and the posterior wall of the bladder (data not shown). Cystoscopy revealed that the bladder volume was reduced and the mucosa at the bladder floor and neck was red (data not shown). The symptoms of EC included acute dysuria, suprapubic pain, frequent urination, urgent urination, urge incontinence and odynuria. Biopsy of the lesions through the urethra revealed infiltration of blood vessels and eosinophils into the muscular layer, accompanied by focal muscle necrosis (data not shown). EC was diagnosed, however there was no eosinophilic proliferation in the bone marrow biopsy (Table II). Anti-inflammatory (Cefmetazole, $100 \mathrm{mg} / \mathrm{kg} /$ day) and cetirizine hydrochloride ( $1 \mathrm{ml} /$ day) treatments were administered for a week. Two months later, the symptoms had disappeared (data not shown). Imaging examinations revealed normal results and the blood eosinophils were normal (Table III). The data which are not presented for case 2 were comparable to the images presented for case 1 .

\section{Discussion}

EC is an uncommon primary inflammatory disorder of the bladder with uncertain etiology. The incidence of EC in male adults is increased compared with female adults. Similarly, the incidence of EC in male children is increased compared with females; the average age of onset in children is 6 years old $(17,18)$. The exact cause of EC remains unclear, however certain studies have suggested that anaphylaxis may be a trigger (5-7). Allergens may include food, dust mites, pollen, condom antigens, iodine and anesthetic creams. Asthma and celiac disease are also associated with EC (8). In the present study, the patient in case 1 was sensitive to 
Table I. Clinical features and manifestations of the disease following admission to hospital.

\begin{tabular}{lccccccccccc}
\hline & & \multicolumn{9}{c}{ Symptoms } \\
\cline { 3 - 6 } Case & Sex & Age (years) & 1 & 2 & 3 & 4 & 5 & Onset time & Allergy history & First diagnosis of EC \\
\hline I & M & 6 & + & - & + & - & + & Acute & Yes & Yes \\
II & M & 7 & - & + & + & + & + & Acute & No & Yes \\
\hline
\end{tabular}

M, male; 1, hematuria; 2, abdominal pain; 3 , irritative symptoms of the bladder; 4, urinary incontinence; 5 , dysuria and urinary retention. + , positive result; -, negative result.
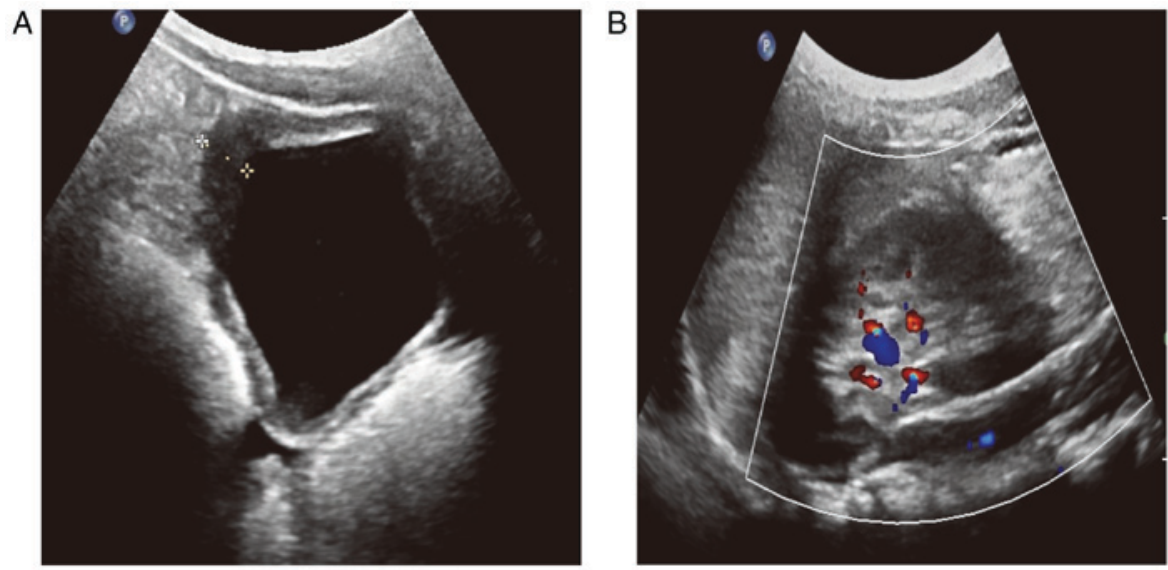

Figure 1. Ultrasonography of the bladder wall of patient 1. (A) Ultrasound revealed irregular thickening of the bladder wall. (B) Color Doppler ultrasound revealed abundant blood flow in the bladder wall. Red indicates flow towards the probe; blue indicates flow away from the probe.
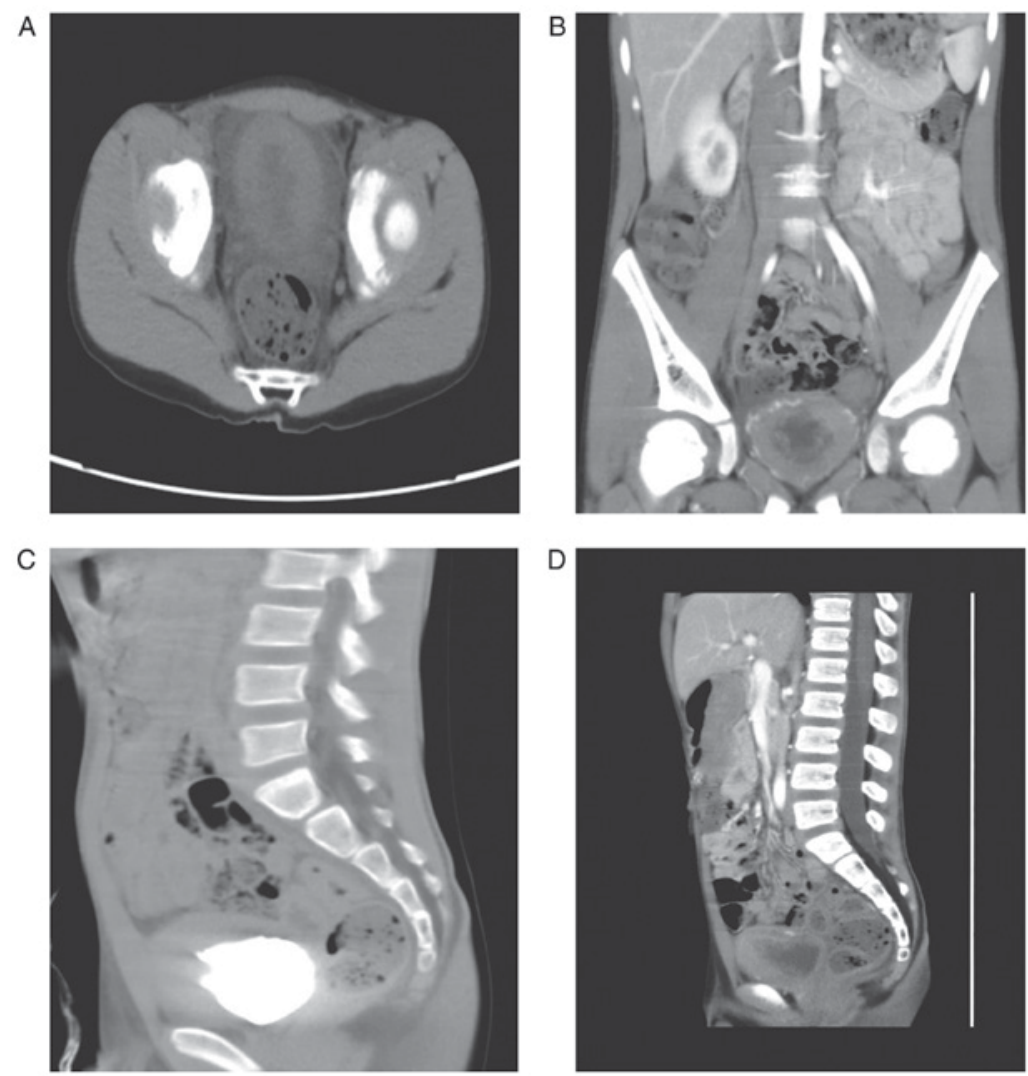

Figure 2. Computed tomography recorded for patient 1. (A) Diffuse thickening and a rough edge were observed in the bladder wall. (B) The volume of the bladder cavity was small and the density of the thickened bladder wall was uneven. (C) Contrast enhanced computed tomography revealed abundant blood flow in the bladder wall and uneven enhancement. (D) The bladder wall was smooth and no obvious filling or defect was observed. 

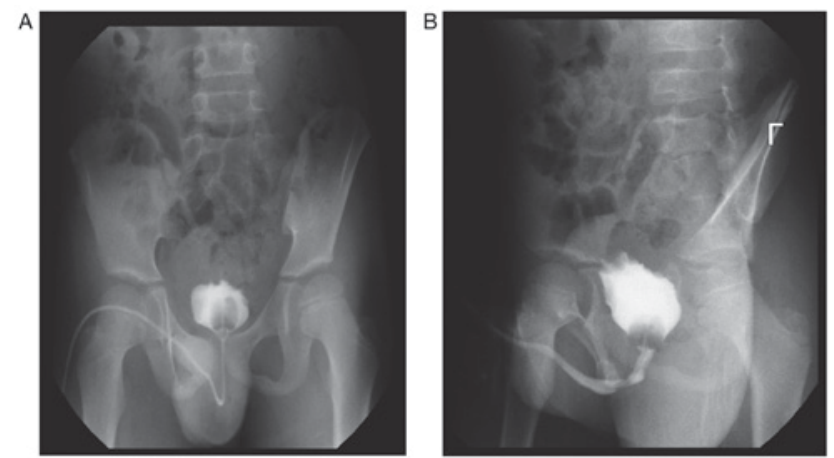

Figure 3. Retrograde angiography of the bladder of patient 1. (A) Front view. (B) Left view. Bladder volume was decreased. The bladder wall was irregular and thickened, with diffusive mucosal lesions.

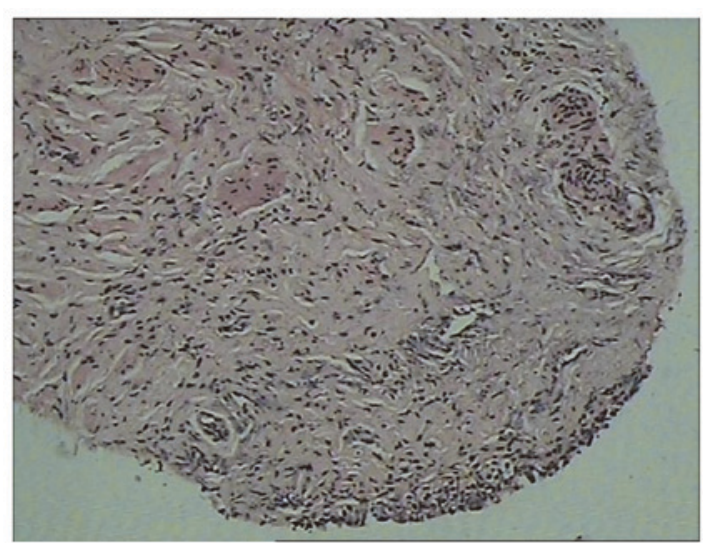

Figure 4. Hematoxylin and eosin staining of the bladder wall tissues obtained from patient 1 (magnification, x100). A small amount of transitional epithelium was observed on the mucosa. Sub-epithelial fibrous hyperplasia was also observed, which was accompanied by inflammatory cell infiltration. The cells were primarily eosinophil granulocytes, however scattered lymphocytes and plasma cells were also observed.

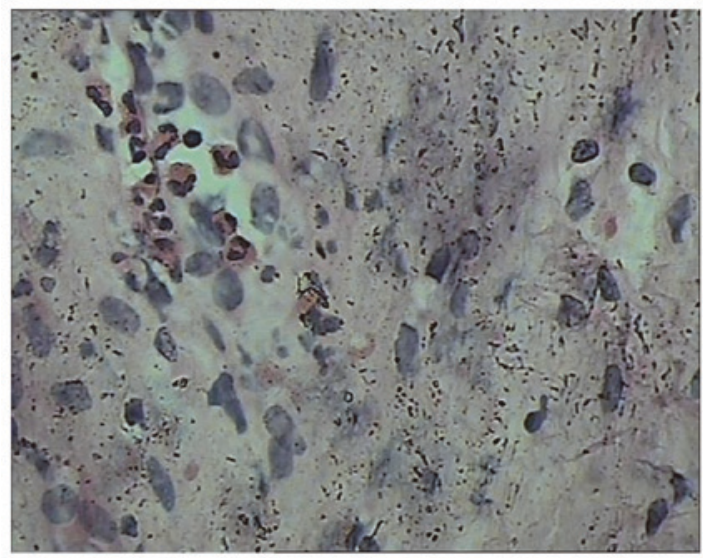

Figure 5. Hematoxylin and eosin staining of the bladder wall tissues obtained from patient 1 (magnification, $\mathrm{x} 400$ ). Eosinophils infiltrated into the right posterior muscular bundle.

Dermatophagoides pteronyssinus and Dermatophagoides culinae, while the patient in case 2 had no specific allergies. The pathogenesis of EC involves IgE-mediated eosinophil activation, with subsequent mast cell degranulation and 
Table III. Treatment, follow-up and prognosis of patients.

\begin{tabular}{llccr}
\hline Case & Treatments & Duration of treatment (weeks) & Follow-up & Prognosis \\
\hline I & $\begin{array}{l}\text { Anti-inflammation, cetirizine hydrochloride and } \\
\text { prednisone }\end{array}$ & 12 & Monthly & Recovered \\
II & Anti-inflammation and cetirizine hydrochloride & 12 & Monthly & Recovered \\
\hline
\end{tabular}

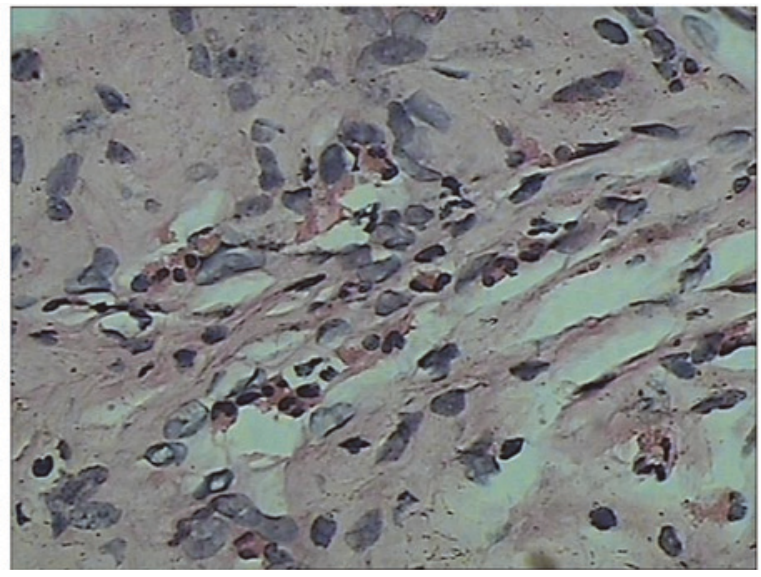

Figure 6. Infiltration of eosinophils into the subepithelial fibrous stroma as observed in patient 1. Magnification, $\mathrm{x} 400$.

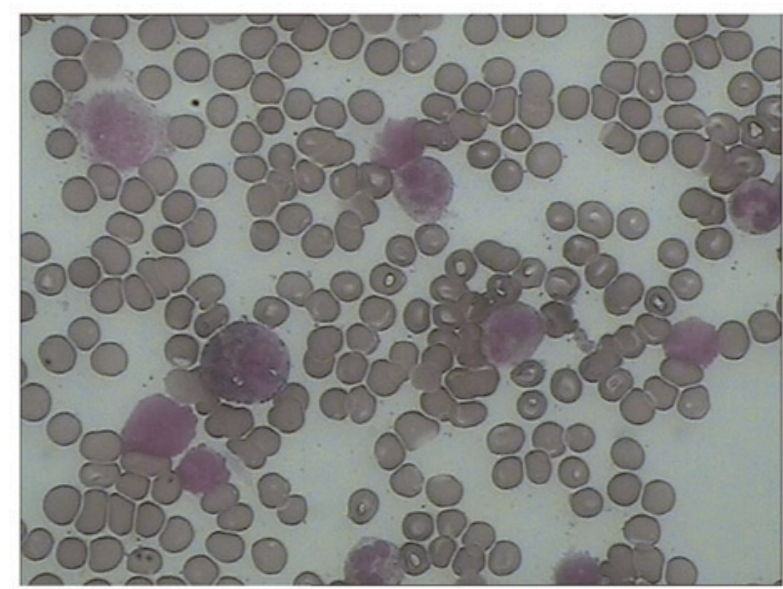

Figure 7. Bone marrow biopsy of patient 1 . Myeloid granulocytic hyperplasia was active, the eosinophil ratio was increased and there was no clear abnormality at each stage. Magnification, $\mathrm{x} 400$.

muscle damage (8). Patients with EC often exhibit a series of urinary symptoms, including frequent urination, hematuria, suprapubic pain, dysuria and daytime and nocturnal enuresis; children with EC may have a clear suprapubic mass (19). The clinical manifestations in the cases presented in the present study were consistent with EC; however, the clinical manifestations of EC are often varied and may be easily confused with nonspecific cystitis (2). Children with suprapubic masses should also be differentiated from those with malignant bladder tumors. EC is a rare condition that can mimic invasive bladder cancer symptoms. EC diagnosis may be considered if a bladder tumor is associated
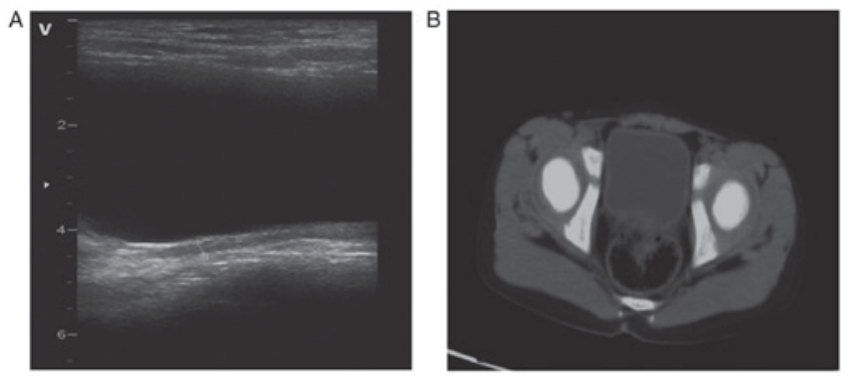

Figure 8. Follow-up with patient 1 at one month post treatment (A) Ultrasonography. (B) Computed tomography. The bladder was well filled. The bladder wall was smooth, even and only slightly thickened. No clear filling or defects were found.
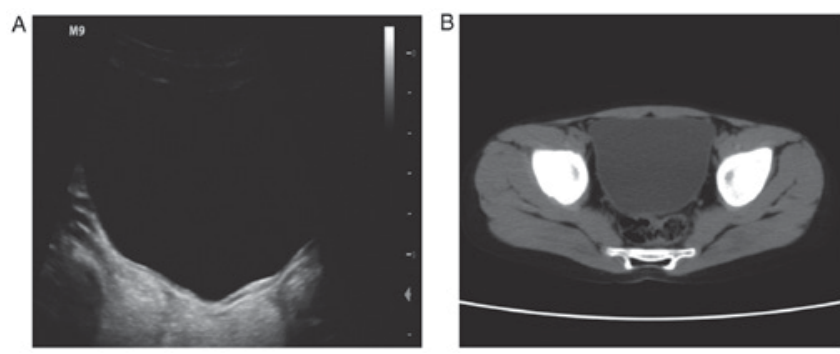

Figure 9. Follow-up with patient 1 at three months post treatment. (A) Ultrasonography. (B) Computed tomography. The bladder wall was smooth, even and not thickened. No filling or defects were observed.

with eosinophilia (20). The eosinophil count in the blood was significantly increased in each of the 2 cases and a bone marrow biopsy revealed increased eosinophils. In a number of patients with EC, peripheral blood eosinophilia occurs without reaching the level of eosinophilia syndrome. Patient 2 only received treatment by anti-inflammatory and cetirizine hydrochloride without steroid therapy. During hospitalization, the eosinophils were found to be decreased in a routine blood test and the patient's symptoms gradually improved. It has previously been suggested that EC may also present a self-healing trend (3). In the present study, patient 1 presented with similar symptoms 3 years earlier; an ultrasonography revealed thickening of the bladder wall and following 3 days of anti-inflammatory treatment, the symptoms disappeared. In addition, patient 1 was allergen-positive. The authors hypothesize that the onset of the disease in case 1 was associated with allergens and that, with the elimination of inflammatory mediators, symptoms may disappear (3). It has previously been reported that urine cultures are positive in EC patients (4). However, the urine cultures in each of the cases presented herein were negative. 
Eosinophils are rarely observed in urine sediments and are rapidly degraded or rarely detached from the mucosa (21). Certain EC patients have hematuria symptoms $(22,23)$. If an imaging examination reveals bladder wall thickening, which is similar to mass infiltration, a tumor is suggested. Cystoscopy results usually suggest bladder rhabdomyosarcoma (6). Intense inflammatory changes, including congestion and edema of the bladder wall, may result in intense inflammatory changes in the bladder wall and associated with this, lesion may produce excrescences, which resemble vesical rhabdomyosarcoma (24). As EC is very rare in children there are no ideal guidelines for its treatment and follow-up and treatment is typically based on experience (25). First-line treatments typically involve the removal of any suspected allergens, followed by the use of antihistamines and corticosteroids. It has been reported that corticosteroids may accelerate the remission of symptoms and stabilize lysosomal membranes due to their anti-inflammatory effects (16). For refractory cases, cyclosporine $A$ is administered orally for 8 months (26). For children with peripheral blood eosinophilia, montelukast sodium is used (27). Researchers have also tried intravesical instillation of dimethyl sulfoxide twice a week (27). EC in children is normally benign and self-limiting, however it may still develop into bladder fibrosis and secondary urinary tract obstruction. The diagnosis and treatment of EC depends on clinical suspicions and histopathological examination.

In conclusion, EC in children is similar to a tumor, however it has its own characteristics. Although it is a rare disease it should be considered when urinary tract symptoms and bladder wall thickening are observed in children. Bladder biopsy and histopathological evaluation are important for the diagnosis of EC and allow for the selection of an appropriate treatment.

\section{Acknowledgements}

The authors would like to thank Dr Li Hong Tan for his support and encouragement. The authors would also like to thank Dr. Feng Ning and Professor Weijian Chen for their technical support and pathological advice.

\section{Funding}

This work was supported by the Natural science foundation of Hunan Province (grant no. 10JJ5042).

\section{Availability of data and materials}

The data analyzed during the current study are available from the corresponding author on reasonable request.

\section{Authors' contributions}

$\mathrm{JuH}, \mathrm{FN}$ and LT analyzed and interpreted data regarding eosinophilic cystitis. JiH obtained imaging data and WC performed histopathological experiments. JuH contributed to writing the manuscript. YZ conceived the study and helped revise the manuscript. All authors have read and approved the final manuscript.

\section{Ethics approval and consent to participate}

Not applicable.

\section{Patient consent for publication}

The patients provided written informed consent for the publication of their data and associated images.

\section{Competing interests}

The authors declare that they have no competing interests.

\section{References}

1. Brown EW: Eosinophilic granuloma of the bladder. J Urol 83: 665-668, 1960

2. Saadi A, Bouzouita A, Ayed H, Kerkeni W, Cherif M, Ben Slama RM, Derouiche A and Chebil M: Pseudotumoral Eosinophilic cystitis. Urol Case Rep 3: 65-67, 2015.

3. Park H: Eosinophilic cystitis with recurrent urinary retention: Case report. Res Rep Urol 9: 51-53, 2017.

4. Runge SB, Høyer S and Winding L: Macroscopic hematuria and a bladder mass: Eosinophilic cystitis in a 7-year-old boy. Case Rep Radiol 2016: 9346218, 2016.

5. Sparks S, Kaplan A, DeCambre M, Kaplan G and Holmes N: Eosinophilic cystitis in the pediatric population: A case series and review of the literature. J Pediatr Urol 9: 738-744, 2013.

6. Li G, Cai B, Song H and Yang Z: Clinical and radiological character of eosinophilic cystitis. Int J Clin Exp Med 8: 533-539, 2015.

7. Ozdoğan EB, Arslansoyu Çamlar S, Bilen S, Imamoğlu M, Tiraş S, Cansu A and Ozoran Y: An unusual cause of terminal hematuria in a child: Eosinophilic cystitis. Can Urol Assoc J 8: E867-E871, 2014.

8. Ladocsi LT, Sullivan B and Hanna MK: Eosinophilic granulomatous cystitis in children. Urology 46: 732-735, 1995.

9. Rossanese M, Palumbo V, Sioletic S, Crestani A, Giannarini G and Ficarra V: Surgical treatment of Eosinophilic cystitis in adults: A report of two cases and a literature review. Urol Int: Mar 19, 2018 (Epub ahead of print).

10. Shaocong Z, Yufeng L, Dao W, Bai L, Shufang S and Linlin W: Eosinophilic cystitis in children: A report of 7 cases and literature review. J Clin Pediatr 35: 304-306, 2017.

11. Zhou AG, Amin A, Yates JK, Diamond DA, Tyminski MM, Badway JA, Ellsworth PI, Aidlen JT and Owens CL: Mass forming Eosinophilic cystitis in pediatric patients. Urology 101: 139-141, 2017.

12. Mosholt KS, Dahl C and Azawi NH: Eosinophilic cystitis: Three cases, and a review over 10 years. BMJ Case Rep 2014: pii: bcr2014205708, 2014.

13. Saad AG: Normal quantity and distribution of mast cells and eosinophils in the pediatric colon. Pediatr Dev Pathol 14: 294-300, 2011.

14. Casanovas M, Fernández-Caldas E, Alamar R and Basomba A: Comparative study of tolerance between unmodified and high doses of chemically modified allergen vaccines of Dermatophagoides pteronyssinus. Int Arch Allergy Immunol 137: 211-218, 2005.

15. Al Johi RS, Seifeldein GS, Moeen AM, Aboulhagag NA, Moussa EM, Hameed DA and Imam HM: Diffusion weighted magnetic resonance imaging in bladder cancer, is it time to replace biopsy? Cent European J Urol 71: 31-37, 2018.

16. Gerharz EW, Grueber M, Melekos MD, Weingaertner K, Barth P and Riedmiller H: Tumor-forming eosinophilic cystitis in children: Case report and review of literature. Eur Urol 25: 138-141, 1994.

17. Thomas JC and Ross JH: Eosinophilic cystitis in a child presenting with a bladder mass. J Urol 171: 1654-1655, 2004.

18. Sujka SK, Fisher JE and Greenfield SP: Eosinophilic cystitis in children. Urology 40: 262-264, 1992.

19. Thijssen A and Gerridzen RG: Eosinophilic cystitis presenting as invasive bladder cancer: Comments on pathogenesis and management. J Urol 144: 977-979, 1990.

20. Werbrouck C, Marrannes J, Verhamme L, Steenkiste E, Laridon E and Van Holsbeeck B: Eosinophilic cystitis mimicking bladder tumor. JBR-BTR 97: 375, 2014. 
21. Popescu OE, Landas SK and Haas GP: The spectrum of eosinophilic cystitis in males case series and literature review. Arch Pathol Lab Med 133: 289-294, 2009.

22. Chia D: Eosinophilic cystitis and haematuria: Case report of a rare disease and common presentation. Int J Surg Case Rep 24: 43-45, 2016

23. Venkatesh KS and Bhat S: Eosinophilic cystitis: A rare cause of hematuria in children. Case Rep Nephrol 2012: 710230, 2012.

24. Redman JF and Parham DM: Extensive inflammatory eosinophilic bladder tumors in children: Experience with three cases. South Med J 95: 1050-1052, 2002.
25. Castillo J Jr, Cartagena R and Montes M: Eosinophilic cystitis: A therapeutic challenge. Urology 32: 535-537, 1988.

26. Aleem S, Kumar B, Fasano MB, Takacs E and Azar AE: Successful use of cyclosporine as treatment for eosinophilic cystitis: A case report. World Allergy Organ J 9: 22, 2016.

27. Zaman SR, Vermeulen TL and Parry J: Eosinophilic cystitis: Treatment with intravesical steroids and oral antihistamines. BMJ Case Rep 2013: pii: bcr2013009327, 2013. 